\title{
Queering the city: Spatiality and territoriality of LGBT lives in the cities of Southern Europe and the Global South
}

Since the 1980s several studies, particularly in Anglo-Saxon and francophone literature, have demonstrated how the settlement and the development of "gay villages" or LGBT neighbourhoods in specific parts of urban contexts around the world (but mostly in North America and Europe) have given rise to specific forms of living the city and have promoted the vitality of certain spaces, more or less open to the rest of the cities in which they are integrated. With similarities to many other areas that have been the main drivers of urban revitalization of innercities, such as cultural and creative quarters or multicultural spaces, these areas are distinguished by the social practices of their users and inhabitants, the specificities of their economic activity, or their contribution to creativity or social integration. More than community ghettos, these areas have been characterized by the coexistence of diverse lifestyles, trajectories and identities, but also by the contribution of LGBT people to gentrification processes through their strong commercial, residential and symbolic presence.

However, this model does not cover the complexity of practices, attitudes and representations that naturally take place in these places, which naturally are not restricted to those areas in those cities, nor does it encompass the diversity and complexity of identities that are inherent to them. Furthermore, these studies report interesting data and results based on empirical work on the spatiality and territoriality of LGBT communities (essentially the most visible ones), but mostly in the Global North (particularly North America and Northern and Central Europe). The importance of these countries' socio-cultural specificities (e.g., in religious, cultural or regulatory terms) in these processes should especially be considered when we question the practices, the representations, the values, and the (in)visibilities of these populations in predominantly Catholic or Muslim countries, for instance. Further studies, taking into account the social, cultural, religious, economic and political diversity of realities such as the ones of Southern Europe and of the Global South, particularly Latin America and Africa, gain pertinence, and must be carried out.

More recently, literature on these topics - particularly originating from the fields of cultural and gender studies and spreading also to other social sciences - has drawn attention to the relevance of queer identities in these processes, emphasizing much more diverse, flexible and complex patterns than the spatialities and the territorialities of LGBT lives take on in contemporary cities, as well as the call for a more attentive eye on the social, cultural and economic practices of these communities. In line with this, the need to look further into the used mechanisms in the negotiation of their identities is also underlined, in order to disentangle the complexity that embodies the individual and collective LGBT life in the city and the reconfiguration of self and group identities in the urban space, from a queer perspective.

This special dossier aimed to shed new light on these topics by questioning spatiality and territoriality of LGBT lives in the cities of Southern Europe and of the Global South, in these multiple perspectives. Among other issues, it called out for debate on key questions such as: queer practices and representations' geographic patterns in the city; specificities regarding personal experiences, negotiations and identity (re)constructions in LGBT neighbourhoods; the importance of 'safe zones' for identity manifestation and everyday practices; spatialities and temporalities in liminary and transgression processes; urban conviviality and nightlife LGBT dynamics; queer performativity and its relation with public space festivalization; the transience/ permanence and visibility/ invisibility dynamics of queer experiences in the urban milieu; and, manifestations of political statement and fight for urban citizenry.

The connection established by culture and creative dynamics with the urban space plays, therefore, a fundamental role in the aesthetics, symbolic and material LGBT presence in the city and the queer prominence in terms of the 
arrangement of certain neighbourhoods or areas, as well as the very redefinition of urbanity. Indeed, artistic production has brought about abundant and substantive contributions, in several national contexts, in terms of subjects who seek to unveil through their work complex features regarding queer subjectivities in the city. Hence, we were also interested in observing the role played by the arts as a disruptive agent, framing the artist as someone who arouses reactions that would otherwise remain hidden behind the social and artistic backdrop. The arts have never set out to appease understandings, nor to conform behaviours; rather, they intend to tease, to surprise, to provoke thoughts and feelings and, perhaps, to pave the way for debate.

About 40 years ago, the Brazilian anthropologist José Carlos Rodrigues (2006) ${ }^{1}$ discussed, in what became a seminal work in his country, certain features of the social construction of the body and its position in different human societies and cultures. According to him, our challenge is to show how the social dimension appropriates the body in a way that (the underlying assumption of an 'increasingly physiological physiology', as well as an 'increasingly anatomical anatomy') has become for the modern man, within the dominant scientism, a sort of naturalization of what is actually relational and symbolic" (p.116); however, this dialogue is still lacking a critical, open, straightforward approach in some societies. For certain bodies and identities, free circulation in urban spaces remains (or is seemingly more) forbidden.

Another development raising our attention are the contemporary types of social control, violence and neoobscurantism within the city. The Brazilian geographer Milton Santos has stated that all territories are political and that understanding their use ultimately enables the comprehension of society itself. In the symbolic domain, the constitution of identities is impacted by the conservative and reactionary shift that is taking place nowadays in urban spaces around the world. Tales of dissent have a very strong territorial bearing, where the arts provoke political debate pushing the boundaries of a certain era. Likewise, there have also been significant impacts stemming from the present-day sociability model and a few obscurantist, violent and conservative movements which have not been able to ward off substantial trends for diversity, tolerance and progress that many spaces (more or less institutionalized, ephemeral or visible) have achieved, becoming more resilient in many cities around the globe.

This debate should then be stimulated, both in the arts and in critical reflection engendered by scientific knowledge, particularly the one produced by the empirical observation of subjects moving through these and other spaces of knowledge production. Our configuration of social knowledge production concerning subjects that challenge established theoretical models should remain open for debate, wherever possible.

Opening this dossier Débora Armelin Ferreira's essay analyses how the language of performance (body art) can be used as a mighty tool towards transsexual artists' empowerment and visibility, reviewing the artwork of Brazilian artist Lyz Parayzo. The author seeks to demonstrate how these productions create space for the construction of her own narratives within the arts field, and within the urban space, as a means of resistance and survival in Sao Paulo, one of Brazil's most violent cities - and also one of the most significant ones in terms of the country's artistic circle. Ferreira demonstrates how Parayzo views her work as an instrument for resistance and fight, as well as defence, against a context of extreme violence for the trans population which includes this artist.

Following this essay, the perspective of art historian Gaudencio Fidélis is presented (curator of the first queer exhibition in Latin America, held in Brazil in 2017) on the emblematic events that followed the opening of the exhibition in the city of Porto Alegre, Brazil. The interview was held in the fall of 2019 in the city of Porto and was focused on the controversies surrounding the exhibition "Queermuseu: cartographies of the difference in Brazilian art", conceived and organized by this Brazilian curator. The objective was to understand, from his point of view as main responsible, the problems generated around the first queer exhibition in Brazil and Latin America. The curator presents details of events that have exposed a conservative and reactionary component of Brazilian society, which in the middle of the $21^{\text {st }}$ century persists as a remarkable characteristic. The interview also underlines the idea that queer theory is a reflection on gender and sexuality. However, it is also an important epistemological tool to question heteronormativity itself, extrapolating the questions to the essentialism of

\footnotetext{
${ }^{1}$ Rodrigues, J.C. (2006) Tabu do Corpo. Rio de Janeiro: Fiocruz. 154 pp. ISBN: 857541-089-X.
} 
binarisms in general. It is in this perspective that important political contributions have been made to social theory and, of course, also to the understanding of urban space.

Marco La Rocca's article presents a case study that seeks to understand how the form of heteronormative colonization emerged, in an effort to recover the intellectual debate on gay consumer spaces. Although this work has thoroughly investigated the progressive 'straight turn' of major gay spots, Rome's Gay Village presents itself as an urban artefact that not only fails to convincingly challenge spatial heteronormativity, but also fails, to some extent, to successfully replicate a classic urban 'gay-friendly' paradigm through consumption. This happens in a metropolitan context fully integrated with the notion of the 'West', even though it is marked by the specificities of Southern Europe. Consequently, Rome's Gay Village challenges, from the inside, the assumptions about the uniformity of the West's geopolitical construction in terms of gender and sexual issues, while at the same time, resonates the academic problematization of urban models that attempt to conjugate queer liberation with capital accumulation. The Gay Village is a three-month summer festival, organized in Rome since 2002 (after the success of the Gay Pride Parade in 2000) that quickly became an important summer event. The ethnographic work in the Gay Village 2017 edition revealed a significant presence of young heterosexual cisgender men among the festival audience, whose experiences and practices were often transformed into aggressive forms of spatial appropriation, producing a sense of discomfort and insecurity among the Queer public.

Closing the dossier, Pedro Costa and Paulo Pires analyse the specificities of two "LGBT neighbourhoods" in Southern Europe, Príncipe Real in Lisbon and Chueca in Madrid. In a comparative perspective with the analysis that Colin Giraud developed in 2014 in Paris (Marais) and Montreal (Village), the authors pinpoint a set of specificities in these cities that can result from the particularities of the "South", compared to the model of the "gay village" usually advanced by international literature on the subject. Aiming to complexify the ways in which the spatiality and territoriality of experiences in "traditional LGBT neighbourhoods" are usually described, the authors point out the difficulty in applying a model of daily living and symbolic insertion in the city in these countries (and possibly in other realities), marked by a logic of sociability and identity construction confined to the "neighbourhood" and political activism on behalf of the "LGBT community", even if it takes on a symbolic centrality for the population and, unequivocally mobilizes the characteristics of "safe spaces" for the expression of collective and individual identities. In societies where the dominance of heteronormativity and the feeble collective affirmation (or social visibility) of the LGBT community seem to be the rule (particularly in the Portuguese case), the strength of the "socio-spatial" structure of the neighbourhood seems to be diminished in the logics of sociability and identity negotiation, with the symbolic territorial capital rapidly mobilized towards more general processes of massification, tourism and gentrification.

The editors recognize how the diversity of these materials produced interesting contributions, both conceptual and empirical, to the theme of this dossier, as well as to the diversity of disciplinary and theoretical approaches to spatial patterns and territorial mechanisms intrinsic to the lives of LGBT people, their practices and identities, and the identities that exist in contemporary urban space. We expect that this may be an initial contribution to the discussion of the theme and that many others will follow, in the context in which their analysis will be pursued.

Good readings!

Pedro Costa

Simone Amorim

Dossier Editors 